\title{
Laser Texturing of NiTi Alloy with Enhanced Bioactivity for Stem Cell Growth and Alignment
}

\author{
Rui Zhou ${ }^{1, *}$, Xizhao Lu², Shengdong Lin $^{1}$ and Tingting Huang ${ }^{1}$ \\ ${ }^{1}$ School of Aerospace Engineering, Xiamen University, 422 Siming South Road, Siming District, \\ Xiamen, Fujian Province, China 361005 \\ ${ }^{2}$ College of Mechanical Engineering and Automation, Huaqiao University, Xiamen, 361021, China \\ *E-Mail: rzhou2@xmu.edu.cn
}

\begin{abstract}
Surface treatment by pulsed laser ablation is applied to fabricate textured surfaces for biomedical metal substrates-NiTi alloys, aiming to guide aligned growth of human stem cells. The effect of anisotropic micro-structures of textured metal surfaces on human cell alignment and adhesion behaviors is investigated at a groove pitch of different sizes. Human mesenchymal stem cells (hMSCs) cultured on NiTi substrates at inter-ridge distances of 30 and $60 \mu \mathrm{m}$ have a highly parallel alignment, with a preferentially aligned direction along the ridges. In contrast, hMSCs cultured on NiTi substrate at inter-ridge distance of $110 \mu \mathrm{m}$ exhibit an alignment off the direction of lasertextured topography. The surfaces of NiTi substrates are found to be partially converted into the oxidized states, which located mostly in the area of grooves resulting in insignificant hydrophobicity. Pulsed laser ablation is proved to be a versatile and environment-friendly approach to pattern metal surfaces with anisotropic structures for controlling cellular alignment and elongation along the ridges with potential applications in bone tissue engineering.
\end{abstract}

DOI: $10.2961 /$ jlmn.2017.01.0005

Keywords: laser ablation, NiTi substrate, surface texturing, stem cell, alignment

\section{Introduction}

Due to significant demanding in biomedical application, bone tissue engineering has been booming in various areas, such as orthopedic surgery, oral and maxillofacial surgery [1-2]. As one of the basic components of bone tissue engineering, scaffold materials have a broad range of applications, triggering great research interest [3]. A large group of researchers makes use of different materials to fabricate scaffolds, such as natural polymer materials, artificial polymer, natural bone materials and NiTi alloys that have been treated [4]. However, critical limitations still exist for these materials. For example, natural polymer lacks enough mechanical strength. It is difficult to be scaled up in production as well as to control the degradation time [5]. Artificial polymer is usually hydrophobic and the attraction to cells is weak [6]. Natural bone materials often result in immune rejection [7]. Apart from these materials, NiTi shape memory alloys have been widely used in orthopedic surgery [8] due to their inherent shape memory properties and superelasticity [9]. The two features account for modulating fracture properties as well as preventing loosening or fretting at the interface of implant construct [10]. NiTi alloys have other fascinating properties, such as biocompatibility, high recovery strain, and low stiffness to facilitate integration [11]. These remarkable superiorities make NiTi alloys as practicable internal fixation materials.

Tissue functions rely largely on the ordered architecture of cellular alignment while it has been proved that versatile surface topography, such as pores, grooves and ridges in micro/nano-scale, can enhance the binding strength of cells at the same time elicit cell alignment and proliferation [12]. In other word, through the interaction with the textured surface structures, the behavior of tissue cells may be tuned. Hence, artificial biomaterial scaffold is designed to create anisotropic geometries to replace nanoscale structures in real organs, thus facilitating cell growth and guiding cell behaviors [13-14]. The surface roughness and chemical composition are both key factors affecting its surface wettability, which can be tuned by varying laser fluence and pattern design. Wettability is one of the most important properties of biomaterials as hydrophilic surfaces generally facilitate cell adhesion and subsequent better bioactivity [15-16].

There are several popular methods for surface treatment. For instance, plasma spraying technique [17] has been used in clinical practice, but the grain size of the coating is coarse with presence of cracks and holes due to process conditions. While sand blasting technique can make the surface rough, it needs additional process to remove surface contaminations. Reactive ion etching (RIE) [18] and machining/electrochemical micromachining (EMM) [19] are also used for surface treatment to fabricate a regular pattern. However, complex experimental setup, contaminations and other backwards are not ignorable [20].

In this study, a versatile and environment-friendly approach method is presented by using pulsed laser ablation to create periodic groove on NiTi substrates. Because of its limited heat affected zone, small materials distortion and environment-friendly properties, laser processing can provide an efficient, convenient and controllable method to structure metal surface with scalable micro/nano-patterns [21]. Ridge width and groove pitch of different sizes can be easily controlled by pattern design. Anisotropic structures are fabricated for controlled cellular alignment along the 
ridges. It demonstrates that the micro-spike structures of laser textured metal surfaces at a groove pitch would affect human cell adhesion and alignment behaviors.

\section{Experimental}

Metal substrate texturing

As the laser intensity reaches a certain level, leading to the significant material vaporization and melting at the substrate surface. Due to the temperature distribution, vaporization happens in the center area of laser irradiation with higher temperature, and melting appears in the surrounding area with the lower temperature. A vapor plume above the substrate surface can be formed. Above a certain intensity, the number of species within the plume increases and interactions between the laser beam and the vapor become important. Subsequently, it results in the ionization of species and the vapor is denoted as plasma. Because of the strong nonlinearity in this interaction, small changes in laser parameters cause strong changes in processing results [22]. For this reason, precise control of the laser processing parameters is important.

During laser ablation, a heat affected zone is generated where molten material is re-solidified. The heat affected zone causes the changes of material properties which affect the subsequent laser ablation performance. In order to reduce the transfer of heat out of the ablation zone, pulsed laser is employed to ablate the material surfaces. In this research, the NiTi surface is patterned by a pulsed fiber laser with a wavelength of $1064 \mathrm{~nm}$. The pulse duration is around $10 \mathrm{~ns}$ and the repetition rate is $100 \mathrm{kHz}$. The beam size is $\sim 20 \mu \mathrm{m}$. A galvo-scanner is used to fabricate gratings on the NiTi surface with a scanning speed of 10 $\mathrm{mm} / \mathrm{s}$ and a laser fluence of $5.73 \mathrm{~J} / \mathrm{cm}^{2}$. A field emission scanning electron microscope (FESEM) is used to characterize the surface morphology. Ridge orientation, inter-ridge-distance, and ridge-length are measured using the built-in functions of NIH ImageJ. The inter-ridge distance is measured by drawing a line perpendicular to the ridges, and is equal to the length of the line divided by the crossed-ridge number.

In this paper, we investigated the effect of patterned surface on human stem cell growth and alignment by varying the groove pitch. The varied groove pitch is 20,50 and $100 \mu \mathrm{m}$ with a fixed ridge width at $10 \mu \mathrm{m}$, respectively.

The element analysis of the processed metal substrate surface is carried out by using the energy dispersive X-ray (EDX) technique, including the control sample without any treatment, treated samples with different grooves at 20, 50 and $100 \mu \mathrm{m}$.

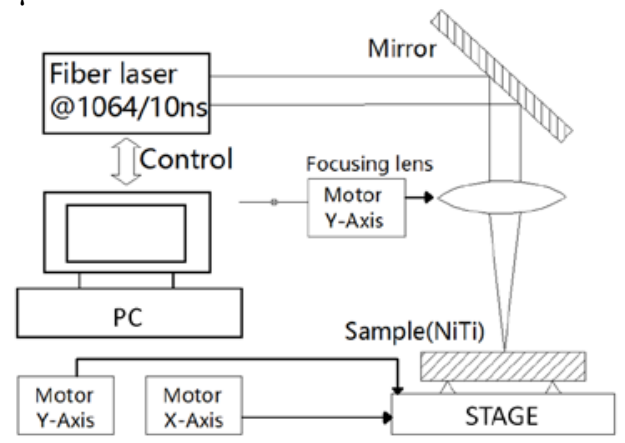

Fig. 1 Experimental setup for laser texturing of NiTi.

\section{Water contact angle measurements}

Water contact angle (WCA) is measured using a video contact angle system (Advanced Surface Technologies; VCA2000). Images are taken $5 \mathrm{~s}$ after drop dispersion to allow full drop development. When a water droplet (drop volume $=2 \mu \mathrm{L}$ ) is deposited on the substrate surface, a high-resolution camera is used to capture the image. The water contact angle is analyzed by a graphic tool of image processing software.

\section{Cell isolation and culture}

Human mesenchymal stem cells (hMSCs) are obtained and used as reported previously [23] and agreed with the international guidelines regarding the use of stem cells for research purpose [24]. hMSCs used in all experiments are within passage 6 (P6) at a constant seeding density of 20,000 per $\mathrm{cm}^{2}$ on the NiTi surface.

\section{Cell alignment and elongation assays}

Cellular alignment and elongation of hMSCs cultured on the textured NiTi substrates are investigated by SEM. Cells after culturing for the pre-determined periods are fixed with $4 \%$ formaldehyde in phosphate-buffered saline (PBS) for 15 mins. After thrice washing with PBS, cells are then dehydrated with a graded series of ethanol-water mixture to pure ethanol and dried in a dry cabinet at $21^{\circ} \mathrm{C}$ for one week.

SEM is employed to evaluate the surface morphology. Images are taken for surface information at a high voltage of $15 \mathrm{kV}$ at different magnifications. For the analysis of morphology consistency, representative areas of textured metal are selected. A $10 \mathrm{~nm}$ thick gold film is deposited on the samples in order to obtain the SEM images with a good quality.

\section{Results and discussion}

\section{Characterization of textured substrate morphology}

Periodic microstructures are fabricated on the NiTi substrates by controlling the laser beam moving along the parallel grooves. Due to the absorption of the laser energy by the NiTi surface materials under the laser irradiation, the targeted substrate is heated up, leading to the melting of surface NiTi and eventually evaporated away.

The overall SEM images in Fig 2 clearly shows the patterns generated by pulsed laser ablation. As can be seen, the boundaries of grooves and ridges are clear and there is no contamination on substrate surfaces. The varied widths of groove pitch are 20, 50 and $100 \mu \mathrm{m}$ respectively by fixing the width of ridge pitch at $10 \mu \mathrm{m}$ constantly, indicating that pulse laser ablation is a reliable, flexible and convenient method to pattern metal surfaces.

As can be seen from the inset shown in Figs 2(a-c), the metal surfaces have almost the similar topographies. It is observed that the surfaces of grooves are rough with a plenty of irregular micro-spike and microporous structures.

Heat-affected zone can change the surface morphology by recasting and formation of burrs. In the laser ablation, compressive forces can be created by the vapor and plasma plume at the laser focus point, leading to expulsion of molten pool of materials [21]. A portion of the liquefied material is resolidified after pressed out of the ablation zone. It is observed that plenty of nano-structures are formed and 

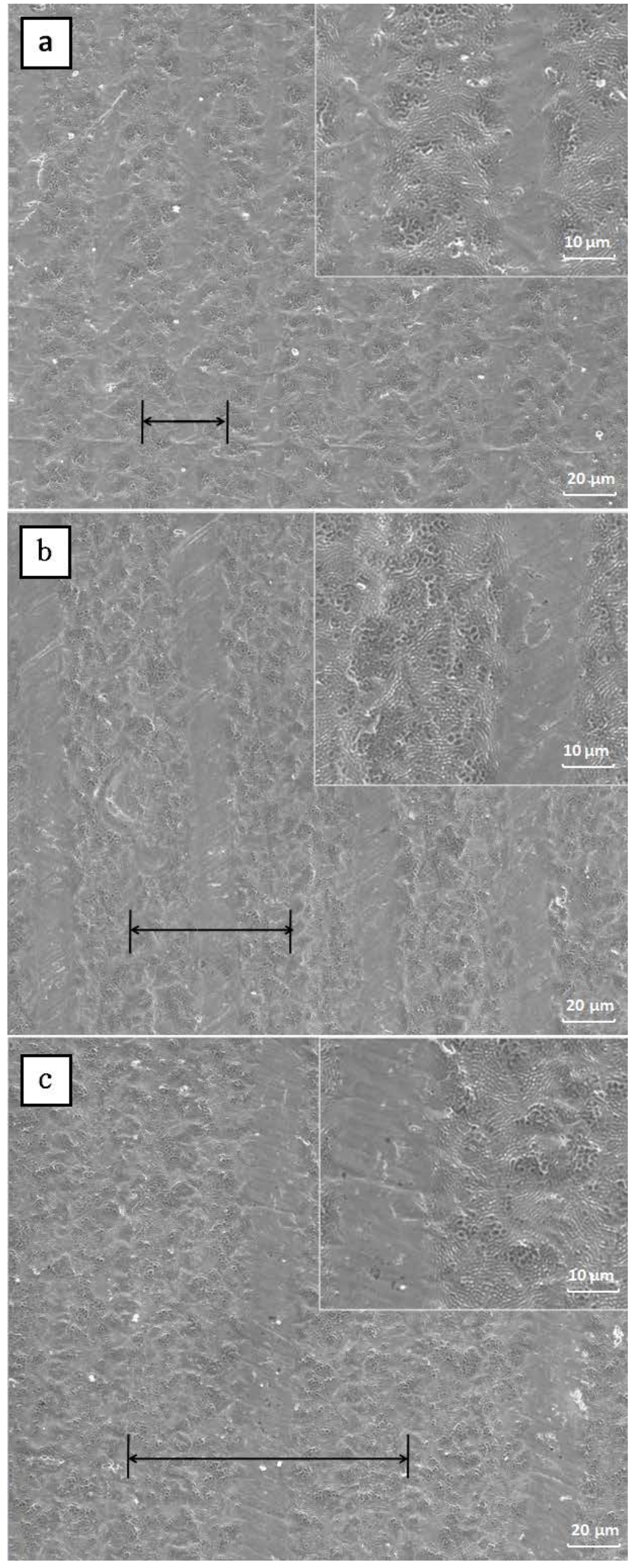

Fig. 2 SEM images of metal surfaces patterned by the pulsed laser at different groove pitches of (a) $20 \mu \mathrm{m}$, (b) $50 \mu \mathrm{m}$ and (c) $100 \mu \mathrm{m}$. The white scale bars in lower magnification regions and insets are $20 \mu \mathrm{m}$ and $10 \mu \mathrm{m}$, respectively. The black bars are the pitches of the central lines of grooves. scattered on the surface of the micro-structures, as shown in the inset in Fig 2. The situation is attributed to Gaussian distribution [25] of the laser beam at the focus points so that the energy density at the border of the laser beam is lower than that of the central part. The surrounding area of laser ablation absorbs less laser energy so that only the center lines of the single-unit groove is melted and formed liquid pools where the heating temperature is not high enough to vaporize the metal. In this process, the ripples are generated on the surfaces of metal liquid pools, forming a large amount of nano-structures since the metal liquid is splashed and shrunk into nanoparticles by resolidification. Nanoparticles redeposited onto the surfaces are the byproduct of the laser ablation. After the laser irradiation, the metal surface layer is cooled down rapidly by heat conduction into the metal substrate. Metal vapor condensates into nanoparticles via strong collisions with ambient gas molecules and finally scatters on the top of the microstructures, which form double-scaled structures on the substrate surface. These nanostructures are in the size of $500 \sim 800 \mathrm{~nm}$.

\section{Characterization of surface chemical compositions}

During the laser ablation, chemical compositions of the substrate materials are changed. Oxygen, water vapor or other gases and contaminants in the ambient air interact with the metals under an environment of a high temperature [26].

Fig 3 shows EDX spectra of the surfaces before and after the laser ablation. It is observed that the distinct oxygen peak appears besides the peaks of nickel and titanium. This is because the nickel and titanium at the surface layer are oxidized during the laser irradiation.

Table 1 shows atomic percentage of NiTi alloy surface. The reference surfaces is composed by nickel and titanium and the average atomic percentage of $\mathrm{Ni}: \mathrm{Ti}$ is about 51:49, there are no other elements. However, oxygen is found from the grooves, and the average atomic percentage of Ni:Ti:O is about 38:39:23. The reason why generating oxygen can be attributed to the heat generated by laser, which makes NiTi substrate oxidized. In the area of ridges, there are also some oxygen elements contributed by the splashed nanoparticles of vaporized metal, leading to the lower atomic percentage of oxygen compared to that in the grooves.

Table 1. Atomic percentage of NiTi alloy surface.

\begin{tabular}{|c|c|c|c|}
\hline Elements & Reference & Grooves & Ridges \\
\hline $\mathrm{Ni}$ & $51 \%$ & $38 \%$ & $46 \%$ \\
\hline $\mathrm{Ti}$ & $49 \%$ & $39 \%$ & $47 \%$ \\
\hline $\mathrm{O}$ & $0 \%$ & $23 \%$ & $7 \%$ \\
\hline
\end{tabular}



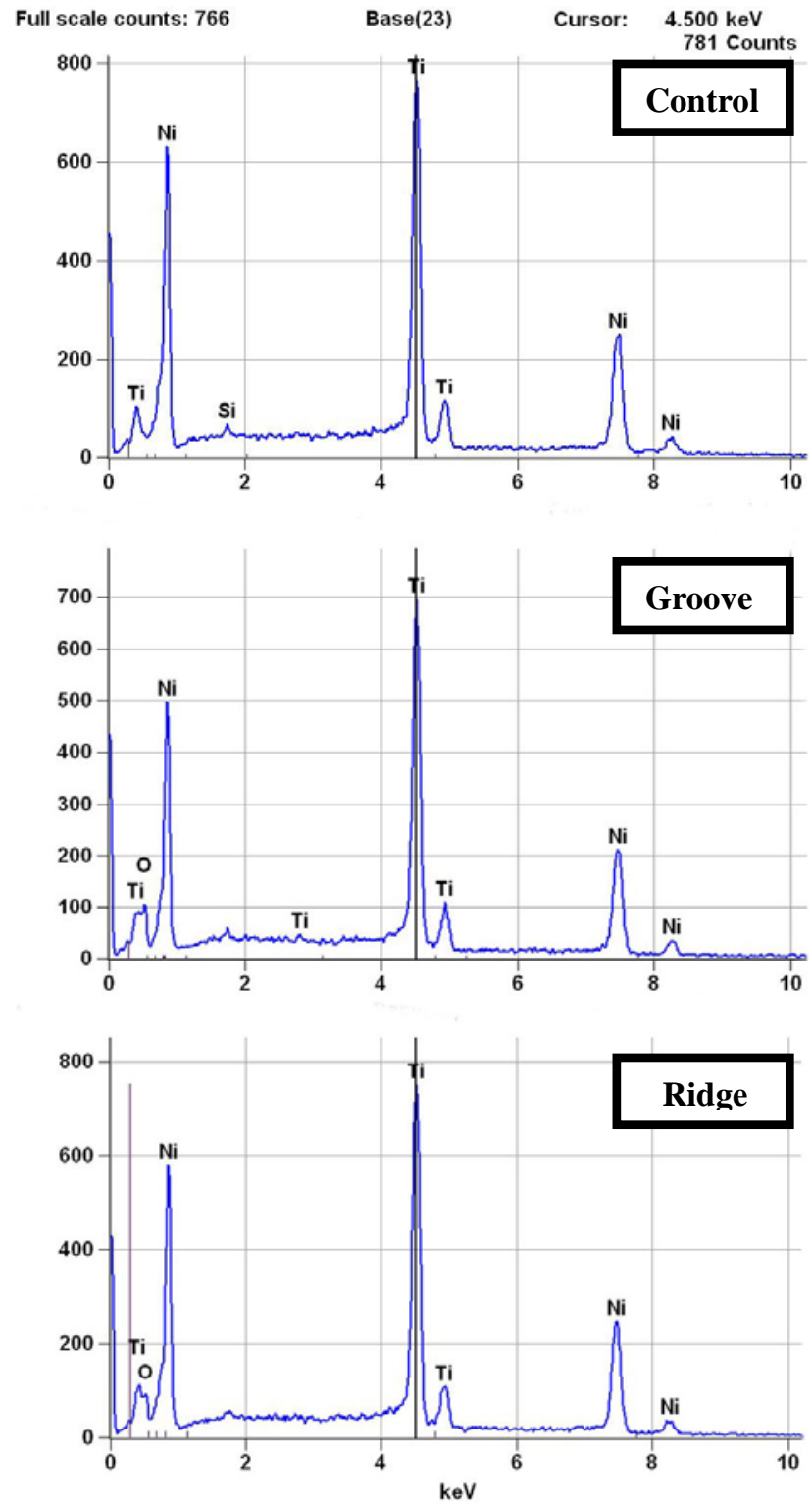

Fig. 3 EDX spectra of metal surfaces in different areas.

By EDX analyses of the surface layer, the atomic percentage of oxygen is approximately one third of the total atomic percentage of nickel and titanium at the grooves and one tenth of that at the ridges. It indicates that the materials of the outer layer on the NiTi surface are only partially converted into the oxidized states of metal alloy with insignificant hydrophobicity.

\section{Surface hydrophilicity}

Fig 4 shows the measured WCA for the NiTi surfaces with different pattern designs. In order to reduce experimental errors, each set of data is measured three times and then averaged. With the decrease of a groove pitch, the contact angle also decreases.

Rough surface can increase the contact angle and lead to super-hydrophobicity. Wenzel's model is dominant at low surface roughness. However, Cassie's model becomes dominant with increasing surface roughness. The contact angle of a water droplet on idealized sinusoidal surfaces is simulated by Johnson [27]. The results show that when the roughness factor is greater than a certain level, the dominant mode could transit from Wenzel to Cassie-Baxter, which reduces the practical contact area between the solid and liquid droplet. In a practical hydrophobic surface with moderate roughness, both modes exist.

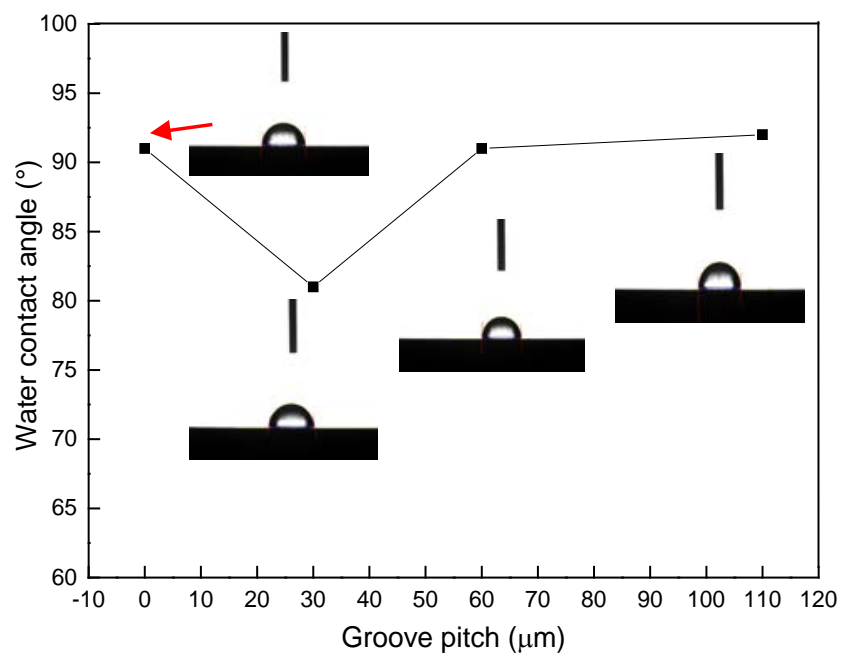

Fig. 4 WCA of NiTi versus groove pitch.

A theoretical consideration is given on the wetting states of dual-roughness surfaces. To adequately describe the contact mode of a water droplet sitting on a surface with two different length scales of roughness, a separate consideration of the contact mode of the liquid with micropillars and nano-pillars should be made. When the water droplet neither wets micron or nano-asperities, it sits on top of the dual scale nano/micron-structures. In this case, water droplet locates upon the microstructures with the air filling inside the microstructures below the droplet, which is governed by Cassie-Baxter model. Cassie-Baxter's equation predicts that the surface roughness further increases the contact angle. Therefore, a higher surface roughness achieves a larger contact angle until it saturates.

\section{hMSC alignment and elongation}

To investigate bioactivity of the textured NiTi substrates for potential bone regeneration, hMSCs are employed and cultured for various periods. As shown in Fig 5, hMSCs exhibit well adhesion to all the laser-textured NiTi surface similar to those of cells on the control group. With prolonged culturing from Day 3 to 5, hMSCs proliferate in all groups of the laser-textured and un-strated substrates. After 5 days of culturing, a more cell confluence of hMSCs can be observed on all the laser-textured NiTi substrates as compared to that of control group. This suggests that the laser-textured topography can enhance hMSCs proliferation, although a similar cell adhesion is obtained on all NiTi substrates at Day 1. 

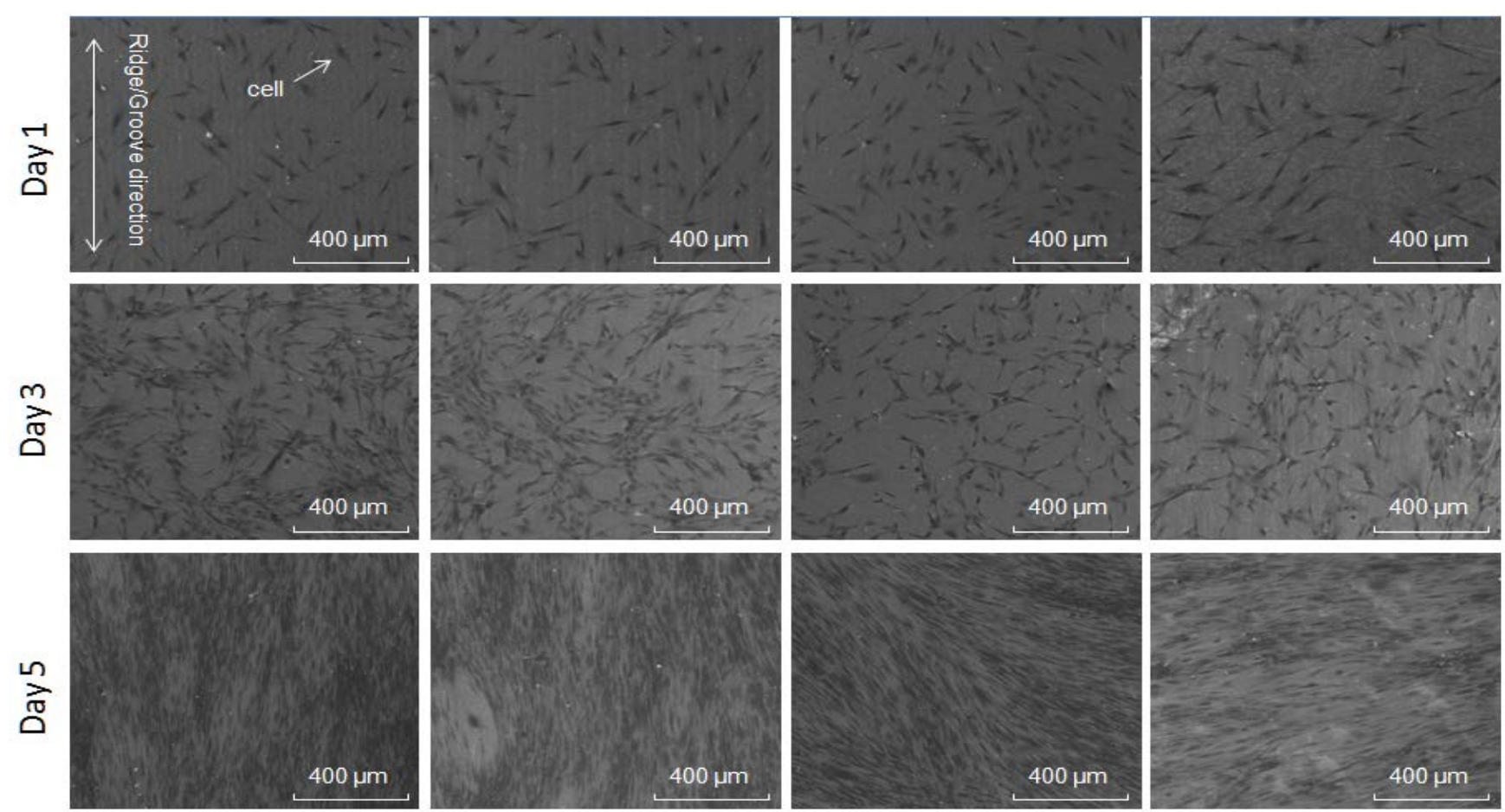

Sample $(30 \mu \mathrm{m})$

Sample $(60 \mu \mathrm{m})$

Sample $(110 \mu \mathrm{m})$

Sample (control)

Fig. 5 Influence of inter-ridge-distance on cellular growth (Scale bar at $400 \mu \mathrm{m}$ : to show cell growth and alignment).
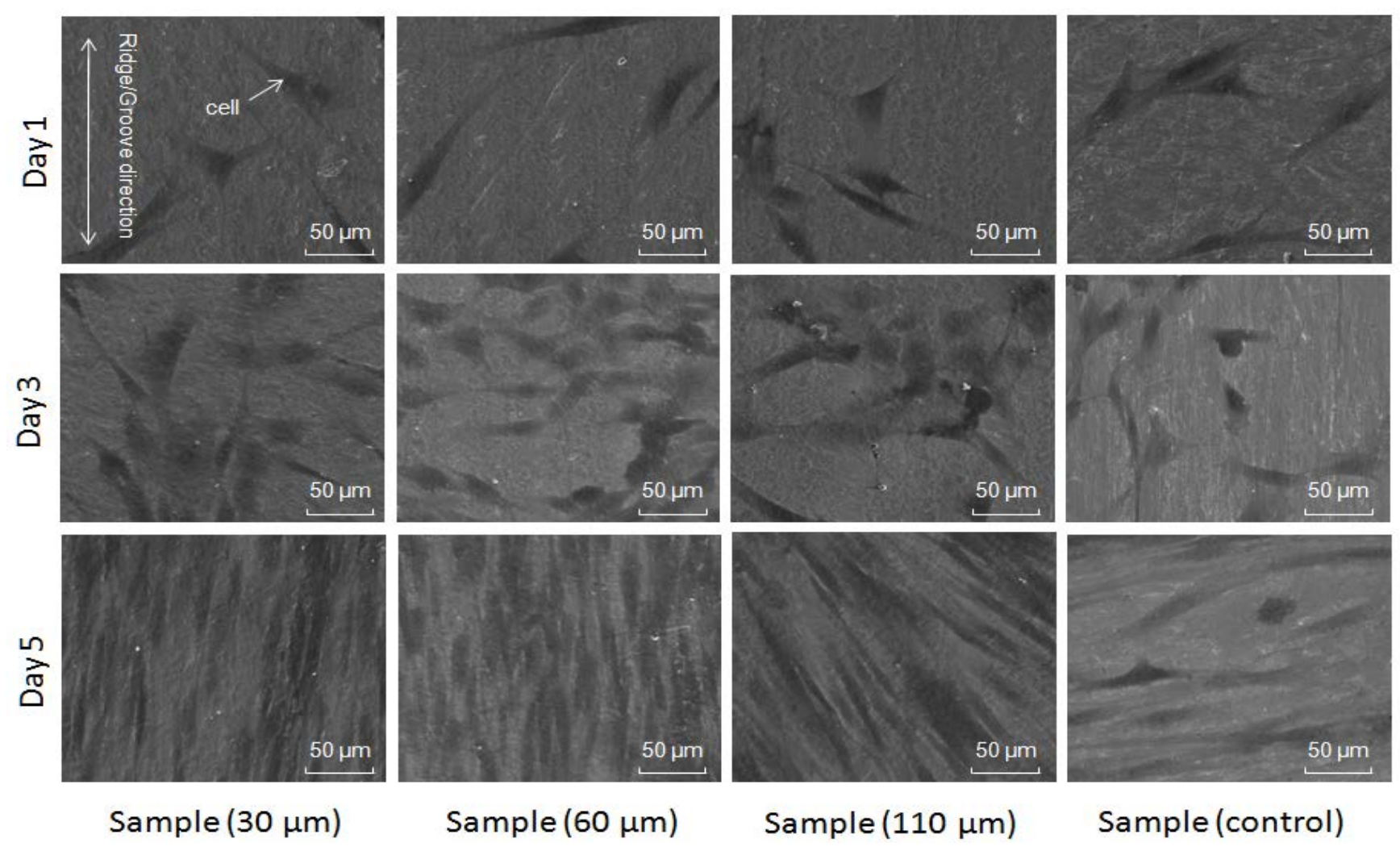

Sample $(110 \mu \mathrm{m})$

Sample (control)

Fig. 6 Influence of inter-ridge-distance on cellular alignment (Scale bar at $50 \mu \mathrm{m}$ : to show cell organization and morphology).

As shown in Fig 6, SEM images show that after 1 day of culturing, hMSCs cultured on surfaces of the lasertextured NiTi substrates exhibit a typical spreading morphology with polygonal appearance. In more instances, these cells exhibit an elongated morphology as that in the native bone tissue, with protrusions extending from the cellular two-ends to adhere onto the substrate surface. With prolonged culturing for 3 and 5 days, most hMSCs adjust 
their morphology into an elongated, spindle-like cell shape as observed from Fig 5. At Day 5, these cells exhibit nearly parallel organization to each other, with a trend to align towards one direction. In contrast, hMSCs of control group have not shown any trend to form alignment.

The effects of anisotropic topography with varied interridge-distance on hMSCs alignment along the lasertextured NiTi topography are then investigated. As shown in Fig 6, hMSCs after 5 days of culturing on NiTi-30 and NiTi-60 show a highly parallel organization, with an alignment, direction preferentially towards the ridges. This organized alignment is found to be uniform at different regions of the whole textured surfaces of NiTi substrates. In contrast, hMSCs cultured on NiTi-110 exhibit an alignment off the direction of laser-textured topography. These results indicate that the laser textured NiTi-30 and 60 have better control on the direction of cell alignment.

\section{Conclusions}

An environment-friendly and versatile approach has been developed to fabricate periodic grooves on NiTi substrates using pulsed laser ablation. Anisotropic microstructures are formed by the dynamic laser ablation. The surfaces of NiTi substrates are partially converted into the oxidized states, and they are located mostly in the area of grooves resulting in insignificant hydrophobicity. Such a laser-fabricated anisotropic topography with varied interridge-distances shows robust modulation on the behavior of hMSCs, with an improvement in cellular proliferation and alignment as compared to those of control groups. Furthermore, hMSCs cultured on NiTi substrates at interridge distance of 30 and $60 \mu \mathrm{m}$ have a highly parallel alignment, with a preferentially aligned direction along the ridges, while cells show an inferior ability to control the alignment direction on the samples at $110 \mu \mathrm{m}$. The laserassisted micro-processing demonstrates a scalable fabrication for patterning NiTi substrates with desirable biofunctions, and has potential applications for bone tissue engineering.

\section{Acknowledgements}

The authors thank the National Key Basic Research Program (2013CBA01703), National Natural Science Foundation of China (61605162), the State Key Laboratory of Optical Technology for Microfabrication of Institute of Optics and Electronics the Chinese Academy of Sciences (Open Project 2016) for financial support.

\section{References}

1. C.M. Agrawal, K.A. Athanasiou, and J.D. Heckman: Porous Materials for Tissue Engineering, 250, (1997) 115.

2. H. Wang, Y. Li, Y. Zuo, J. Li, S. Ma, and L. Cheng: Biomaterials, 28, (2007) 3338.

3. F.R.M. Huss, J.P.E. Junker, J. Hans, and K. Gunnar: J. Plast. Reconstr. Aesthet. Surg., 60, (2007) 543.
4. H. Schliephake, K. Redecker, and T. Kage: Mund Kiefer Gesichtschirurgie, 1, (1997) 115.

5. M. I. Sabir, X. Xu, and L. Li: Journal of Materials Science, 44, (2009) 5713.

6. K.J. Burg, S. Porter, and J.F. Kellam: Biomaterials, 21, (2000) 2347.

7. S.L. Ishaug, R.G. Payne, M.J. Yaszemski, T.B. Aufdemorte, R. Bizios, and A.G. Mikos: Biotechnology and Bioengineering, 50, (1996) 443.

8. R.W.Y. Poon, K.W.K. Yeung, X.Y. Liu, P.K. Chu, C.Y. Chung, W.W. Lu, K.M.C. Cheung, and D. Chan: Biomaterials, 26, (2005) 2265.

9. S. A. Shabalovskaya: Bio-Med. Mater. Eng., 12, (2002) 69.

10. C.W. Muller, R. Pfeifer, T. El-Kashef, C. Hurschler, D. Herzog, M. Oszwald, C. Haasper, C. Krettek, and T. Gosling: Journal of Orthopaedic Research, 28, (2010) 1671.

11. A. Bansiddhi, T.D. Sargeant, S.I. Stupp, and D.C. Dunand: Acta Biomater., 4, (2008) 773.

12. M.J. Dalby, M.O. Riehle, S.J. Yarwood, C.D.W. Wilkinson and A.S.G. Curtis: Exp. Cell Res., 284, (2003) 274.

13. N. Zagris: Micron, 32, (2001) 427.

14. B.D. Ratner, and S.J. Bryant: Annu. Rev. Biomed. Eng., 6, (2004) 41.

15. H. Liao, A.S. Andersson, D. Sutherland, S. Petronis, B. Kasemo, and P. Thomsen: Biomaterials, 24, (2003) 649.

16.X. Liu, and P.X. Ma: Annals of Biomedical Engineering, 32, (2004) 477.

17. P. Fauchais: J. Phys. D: Appl. Phys., 37, (2004) R86.

18. C.M.J.M. Pypen, D. Plenk Jr, M,F, Ebel, R. Svagera, and J. Wernisch: Journal of Materials Science: Materials in Medicine, 8, (1997) 781.

19. C. Liang, X. Yang, Q. Wei, and Z. Cui: Applied Surface Science, 255, (2008) 515.

20. M. Kock, V. Kirchner, and R. Schuster: Electrochimica Acta, 48, (2003) 3213.

21. M. Tang, V. Shim, Z. Y. Pan, Y. S. Choo, and M. H. Hong: J. Laser Micro Nanoeng., 6, (2011) 6.

22. V. Burakov, N. Tarasenko, and N. Savastenko: Spectrochimica Acta Part B: Atomic Spectroscopy, 56, (2001) 961.

23. Z.Y. Wang, E.Y. Teo, M.S.K. Chong, Q.Y. Zhang, J. Lim, Z.Y. Zhang, M.H. Hong, E.S. Thian, J.K.Y. Chan, and S.H. Teoh: Tissue Engineering Part C: Methods, 19, (2013) 538.

24. Z.Y. Zhang, S.H. Teoh, M.S. Chong, J.T. Schantz, N.M. Fisk, M.A. Choolani, and J. Chan: Stem Cells, 27, (2009) 126.

25. Z. Wang, Z. Du, J.K.Y. Chan, S.H. Teoh, E.S. Thian, and M. Hong: ACS Biomaterials Science \& Engineering, 1, (2015) 1239.

26. K. Yamanouchi: Science, 295, (2002) 1659.

27. R.E. Johnson Jr, and R.H. Dettre: The Journal of Physical Chemistry, 68, (1964) 1744. 